\title{
THE SUM OF A DIGITADDITION SERIES
}

\author{
KENNETH B. STOLARSKY
}

\begin{abstract}
Let $B(x)$ be the number of ones in the binary expansion of $x$. A "digitaddition series" is a sequence $y_{1}<y_{2}<y_{3}<\ldots$, where $y_{1}$ is a given positive integer and $y_{n+1}=y_{n}+B\left(y_{n}\right)$ for $n=1,2, \ldots$ Various questions involving the $y_{m}$ are studied; in particular, the asymptotic result $y_{m}$ $\sim(m \log m) /(2 \log 2)$ is proved.
\end{abstract}

1. Introduction. For positive integers $x$, let $B(x)$ denote the sum of the digits in the binary expansion of $x$. For example, the binary expansion of 13 is 1101 , so $B(13)=3$. A sequence of integers $y_{1}<y_{2}<y_{3}<\ldots$ is called a "digitaddition series" if

$$
y_{n+1}=y_{n}+B\left(y_{n}\right), \quad n=1,2, \ldots
$$

Such series have been studied by Kaprekar [7], [11]-[14] and others [1]-[10], [15]-[18]. Much attention [7], [10]-[14], [17]-[18] has been given to selfnumbers, the integers that are not of the form $x+B(x)$. However, the asymptotics of digitaddition series seem to have been neglected. M. Gardner [7] points out (for the corresponding problem in base ten) that no simple formula seems to be known for the sum

$$
S(n)=S\left(n ; y_{1}\right)=\sum_{m=1}^{n} y_{m}
$$

We prove

$$
S(n) \sim\left(n^{2} / 4\right)(\log n) /(\log 2),
$$

and in fact a bit more. We remark that the right side of (1.3) is independent of $y_{1}$. Here $f(n) \sim g(n)$ has the usual meaning, that $\lim f(n) / g(n) \rightarrow 1$ as $n \rightarrow \infty$.

We first show that the sequence $y_{m}$ grows "slowly" by obtaining a crude upper bound for $y_{m}$. Next, we note that if $x$ is a "typical" integer, then $B(x)$ is approximately $\left(\log _{2} x\right) / 2$. Thus, since the sequence $y_{m}$ grows "slowly", most of its terms must be "typical" integers, and hence $y_{m}$ is approximately $\sum_{x=1}^{m}\left(\log _{2} x\right) / 2 \sim\left(m \log _{2} m\right) / 2$. To carry out the details we use the inequality

$$
\sum_{j>(T / 2)+\lambda}\left(\begin{array}{l}
T \\
j
\end{array}\right)<2^{T} \exp \left(-2 \lambda^{2} / T\right) ;
$$

see $[6$, p. 17] or [5].

Received by the editors July 25, 1975.

AMS (MOS) subject classifications (1970). Primary 10A35, 10A40, 10 L99.

Key words and phrases. Binary expansion, digitaddition series, self-number.

(c) American Mathematical Society 1976 
2. The results. Henceforth, $\log t$ shall denote the logarithm of $t$ to the base 2.

THEOREM 1.

$$
S(n)=\left(n^{2} / 4\right) \log n+O\left\{n^{2}(\log n \log \log n)^{1 / 2}\right\} .
$$

Since

$$
\int x \ln x d x=\left(x^{2} / 2\right) \ln x-\left(x^{2} / 4\right)+C,
$$

Theorem 1 can be deduced easily from the following result.

THEOREM 2.

$$
y_{m}=(m / 2) \log m+O\left\{m(\log m \log \log m)^{1 / 2}\right\} .
$$

In particular, $y_{m} \sim(m / 2) \log m$.

3. The proof. We first obtain a crude upper bound on $y_{m}$. Iteration of (1.1) yields

$$
y_{m+1}=y_{1}+\sum_{k=1}^{m} B\left(y_{k}\right) .
$$

The trivial bound $B(x) \leqslant 1+[\log x]$, where $[z]$ denotes the greatest integer in $z$, yields

$$
y_{m+1} \leqslant y_{1}+m+\log \left(y_{1} y_{2} \cdots y_{m}\right) .
$$

The trivial bound $B(x) \leqslant x$, together with (1.1), yields $y_{m} \leqslant 2^{m} y_{1}$. Thus, from (3.2), we find that

$$
y_{m+1} \leqslant m^{2}
$$

for $m$ sufficiently large, say $m>M$. By (3.3) and (3.2) again, we obtain

$$
y_{m+1} \leqslant y_{1}+m+\log \left(y_{1} \cdots y_{M}\right)+\log (m !)^{2} \leqslant 3 m \log m
$$

for $m$ sufficiently large, say $m \geqslant m_{0}$.

We now refine this upper bound. Choose $t$ real so that

$$
[t / \log t]=m \text {. }
$$

Then for $m \geqslant m_{0}$ we have from (3.4) and (3.5) that

$$
1 \leqslant y_{i} \leqslant y_{m} \leqslant 3 t \text { for } 1 \leqslant i \leqslant m .
$$

Next, set $T=1+[\log 3 t]$ and let $\lambda$ be a positive real number. Define $u=u(\lambda)$ by

$$
u=T / 2+\lambda \text {. }
$$

Let $s=s(t, \lambda)$ denote the number of integers $y$ such that $1 \leqslant y \leqslant 3 t$ and

$$
B(y) \geqslant u \text {. }
$$

The number of $y$ such that $0 \leqslant y \leqslant 3 t$ and $B(y)=j$ is at most $\left(\begin{array}{l}T \\ j\end{array}\right)$, so by (1.4) we have 


$$
s \leqslant \sum_{j>u}\left(\begin{array}{c}
T \\
j
\end{array}\right)<6 t \exp \left\{-2 \lambda^{2} / T\right\} .
$$

Now choose

$$
\lambda=(T / 2)^{1 / 2}\left\{\log \left(\log ^{2} t\right)\right\}^{1 / 2} .
$$

Thus

$$
s<6 t / \log ^{2} t
$$

and from (3.1) we have

$$
\begin{aligned}
y_{m} \leqslant & y_{1}+u\{m-1-s\}+T s \\
= & y_{1}+\left\{\frac{\log t}{2}+O\left(\{\log t \log \log t\}^{1 / 2}\right)\right\} \\
& \cdot\left\{\frac{t}{\log t}+O\left(\frac{t}{\log ^{2} t}\right)\right\}+O\left(\frac{t}{\log t}\right) .
\end{aligned}
$$

We conclude that

$$
y_{m} \leqslant t / 2+O\left(t(\log t)^{-1 / 2}(\log \log t)^{1 / 2}\right) .
$$

From (3.5) it is easy to obtain

$$
m \log m \leqslant t \leqslant m \log m+O(m \log \log m) .
$$

Hence

$$
y_{m} \leqslant(m / 2) \log m+O\left(m(\log m \log \log m)^{1 / 2}\right) .
$$

We now use the same method to obtain a lower bound for $y_{m}$. This time define $u$ by

$$
u=T / 2-\lambda
$$

and let $s=s(t, \lambda)$ be the number of integers $y$ such that $1 \leqslant y \leqslant 3 t$ and

$$
B(y) \leqslant u .
$$

Then (note that $\left.\left(\begin{array}{c}T \\ j\end{array}\right)=\left(\begin{array}{c}T \\ T-j\end{array}\right)\right)$ we have

$$
s \leqslant \sum_{j<u}\left(\begin{array}{l}
T \\
j
\end{array}\right)<6 t \exp \left\{-2 \lambda^{2} / T\right\} .
$$

By choosing $\lambda$ exactly as before, we obtain

$$
\begin{aligned}
y_{m} & \geqslant u\{m-1-s\} \\
& =\left\{\frac{\log t}{2}+O\left(\{\log t \log \log t\}^{1 / 2}\right)\right\}\left\{\frac{t}{\log t}+O\left(\frac{t}{\log ^{2} t}\right)\right\} .
\end{aligned}
$$

We conclude from (3.19) and (3.14) that

$$
y_{m} \geqslant t / 2+O\left(t(\log t)^{-1 / 2}(\log \log t)^{1 / 2}\right)
$$

and 


$$
y_{m} \geqslant(m / 2) \log m+O\left(m(\log m \log \log m)^{1 / 2}\right) .
$$

This completes the proof.

4. Remarks. Theorem 2 cannot be improved to

$$
y_{m}=\frac{m}{2} \log m+O\left(\frac{\log m}{\log \log m}\right) .
$$

We also remark that the second difference of $y_{m}$ is unbounded from below. In fact, the inequality

$$
y_{m+1}-2 y_{m}+y_{m-1} \leqslant-\log m+4 \log \log m
$$

holds infinitely often. Both of these assertions are easy consequences of the fact that when the digitaddition series goes past $2^{n}-1$, the number of ones in the binary representations of the $y_{m}$ drops precipitously. We omit the details. Much more than the negation of $(4.1)$ is proved below.

Some open questions: (1) Is $\left|y_{m}-(m / 2) \log m\right| / m$ unbounded? (2) Is $B\left(y_{m+1}\right)-B\left(y_{m}\right)$ unbounded from above as $m \rightarrow \infty$ ? (3) Does the second difference of a digitaddition sequence attain every integer value infinitely often? It is also of interest to determine whether the answers to these questions depend on the choice of $y_{1}$. It is conceivable [2], [3], [8] that for any two digitaddition sequences $y_{1}<y_{2}<\ldots$ and $y_{1}^{\prime}<y_{2}^{\prime}<\ldots$ there exists an integer $k$ depending only on $y_{1}$ and $y_{1}^{\prime}$ such that $y_{n+k}^{\prime}=y_{n}$ for $n$ sufficiently large.

In connection with question (1) we remark that the error term of Theorem 2 is in fact $\Omega\left(m^{1-\varepsilon}\right)$ for any $\varepsilon>0$. This was pointed out by Paul Erdös; the main idea of its demonstration which follows is also due to Professor Erdös.

The proof of Theorem 2 is valid, with no essential change, for any recursion of the form

$$
y_{n+1}=y_{n}+B\left(y_{n}\right)+E\left(y_{n}\right)
$$

provided $E(x)=O\left[(\log x \log \log x)^{1 / 2}\right]$. We only need this fact for $E(x)$ $\equiv 1$. For $\varepsilon>0$ and $n$ large, define

$$
k=\left[n^{-1} 2^{n(1-\varepsilon)}\right] \text { and } m=\left[n^{-1} 2^{n+1}\left(1+n^{-0.1}\right)\right] .
$$

A direct application of Theorem 2 yields

$$
2^{n}<y_{m}<y_{1.1 m}<2^{n+1} \text {. }
$$

Thus for $h<.1 m$ we have that $y_{m+h}=2^{n}+z_{h}$ where $y_{m}=2^{n}+z_{0}$ and

$$
z_{h+1}=z_{h}+B\left(z_{h}\right)+1 \quad(h \geqslant 1) .
$$

Assume that Theorem 2 is valid with an error term $O\left(m^{1-\varepsilon}\right)$. Then

$$
\begin{aligned}
y_{m+k}-y_{m} & =((m+k) / 2) \log (m+k)-(m / 2) \log m+O\left(m^{1-\varepsilon}\right) \\
& >(k / 2) \log m+O\left(m^{1-\varepsilon}\right) \\
& =\frac{1}{2} 2^{n(1-\varepsilon)}+O\left(2^{n(1-\varepsilon)} n^{-1+\varepsilon}\right) .
\end{aligned}
$$

But by the theorem itself, 


$$
\begin{aligned}
y_{m+k}-y_{m} & =z_{k}=(k / 2) \log k+O\left(k(\log k)^{3 / 4}\right) \\
& =((1-\varepsilon) / 2) 2^{n(1-\varepsilon)}+O\left(2^{n(1-\varepsilon)} n^{-1 / 4}\right),
\end{aligned}
$$

and this contradicts (4.7).

In connection with question (3), we remark that if $y_{1}=n$, then the sequence of second differences begins with $g(n)$, where

$$
g(n)=B(n+B(n))-B(n),
$$

and that we have the following

Proposition. Given an integer $a$, there are infinitely many positive integers $n$ such that $g(n)=a$.

Proof. If $a=0$ let $n=2^{q}+2$ where $q \geqslant 3$. If $a \geqslant 1$, set $p=2^{a}-1$ and $n=2^{m_{1}}+\cdots+2^{m_{p-1}}+2^{p}$ where $m_{1}>m_{2}>\cdots>m_{p-1}>p$. If $a<0$ set $q=|a|+1, p=2^{q}-q, r=2 q$, and $n=2^{m_{1}}+\cdots+2^{m_{p}}+2^{r}-2^{q}$ where $m_{1}>m_{2}>\cdots>m_{p}>r$.

\section{REFERENCES}

1. N. Agronomof, Problem 4421, Interméd. Math. 21 (1914), 147.

2. A. Auric, Interméd. Math. 22 (1915), 215.

3. H. Brocard, Interméd. Math. 22 (1915), 134-135.

4. A. Cunningham and others, Problem 12074, Math. Quest. Educ. Times (2) 21 (1912), 52-53.

5. H. Chernoff, A measure of asymptotic efficiency for tests of a hypothesis based on the sum of observations, Ann. Math. Statist. 23 (1952), 493-507. MR 15, 241.

6. P. Erdös and J. Spencer, Probabilistic methods in combinatorics, Academic Press, New York; Akad. Kiadó, Budapest, 1974.

7. M. Gardner, Mathematical games, Sci. Amer. 232 (1975), 113-114.

8. A. Gérardin, Sphinx-Oedipe 1 (1906), 19, 47-48.

9. R. Goormaghtigh, A bit of numerology, Scripta Math. 15 (1949), 91.

10. V. S. Joshi, $A$ note on self-numbers, Math. Student 39 (1971), 327-328 (1972). MR 48 \#8371.

11. D. R. Kaprekar, Cycles of recurring decimals. Vols. I, II, Published by the author, 311 Devlali Camp, Devlali, India, 1953. MR 14, 247; 15, 506.

12. _ , Self-numbers, Scripta Math. 22 (1956), 80-81.

13. _ Puzzles of the self-numbers, Published by the author, 311 Devlali Camp, Devlali, India, 1959. MR 20 \#6381.

14. - The mathematics of the new self-numbers, Published by the author, 311 Devlali Camp, Devlali, India, 1963.

15. A. Makowski, On Kaprekar's "junction numbers", Math. Student 34 (1966), 77 (1967), MR 36 \#6340.

16. A. Narasinga Rao, On a technique for obtaining numbers with a multiplicity of generators, Math. Student 34 (1966), 79-84 (1967). MR 37 \#147.

17. B. Recamán S., Solution to problem E 2408 [1973, 434], Amer. Math. Monthly 81 (1974), 407.

18. A. M. Vaidya, On Kaprekar's tests for self numbers, Math. Student 37 (1969), 212-214. MR $42 \# 186$.

Department of Mathematics, University of Illinois, Urbana, IllinoIs 61801 